This item was submitted to Loughborough's Research Repository by the author.

Items in Figshare are protected by copyright, with all rights reserved, unless otherwise indicated.

\title{
The branch structure of embedded trapped modes in two-dimensional
} waveguides

\author{
PLEASE CITE THE PUBLISHED VERSION \\ LICENCE
}

CC BY-NC-ND 4.0

\section{REPOSITORY RECORD}

Mclver, M., C.M. Linton, and J. Zhang. 2019. "The Branch Structure of Embedded Trapped Modes in Twodimensional Waveguides”. figshare. https://hdl.handle.net/2134/764. 
This item was submitted to Loughborough's Institutional Repository (https://dspace.lboro.ac.uk/) by the author and is made available under the following Creative Commons Licence conditions.

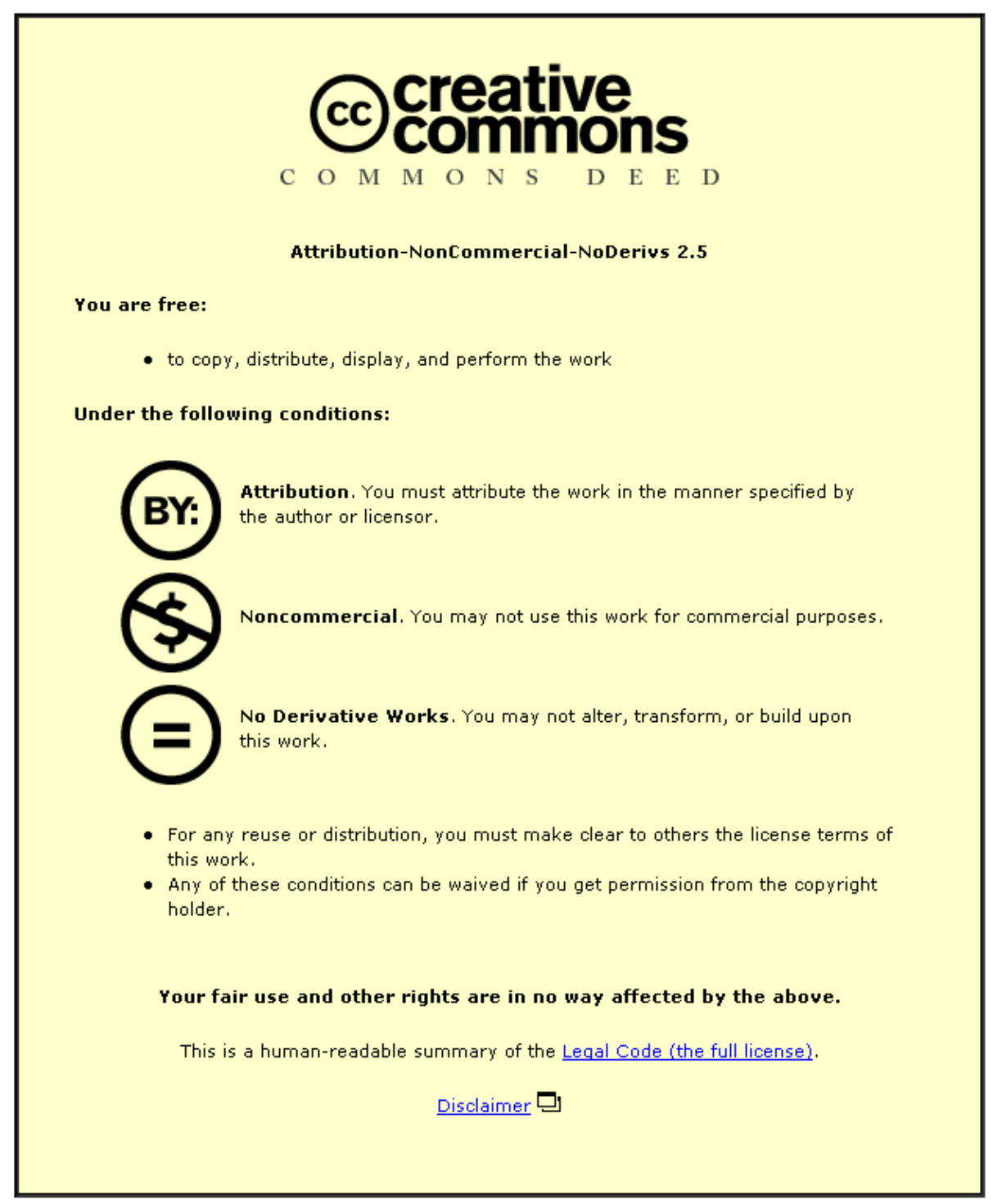

For the full text of this licence, please go to: http://creativecommons.org/licenses/by-nc-nd/2.5/ 


\title{
The branch structure of embedded trapped modes in two-dimensional waveguides
}

\author{
M. McIver, C.M. Linton \& J. Zhang \\ Department of Mathematical Sciences, Loughborough University, Loughborough, Leics., \\ LE11 3TU, UK
}

\begin{abstract}
In this paper we investigate the existence of branches of embedded trapped modes in the vicinity of symmetric obstacles which are placed on the centreline of a twodimensional acoustic waveguide. Modes are sought which are antisymmetric about the centreline of the channel and which have frequencies that are above the first cut-off for antisymmetric wave propagation down the guide. In previous work [1], a procedure for finding such modes was developed and it was shown numerically that a branch of trapped modes exists for an ellipse which starts from a flat plate on the centreline of the guide and terminates with a flat plate perpendicular to the guide walls. In this work we show that further branches of such modes exist for both ellipses and rectangular blocks, each of which starts with a plate of different length on the centreline of the guide. Approximations to the trapped mode wave numbers for rectangular blocks are derived from a two-term matched eigenfunction expansion and these are compared to the results from the numerical scheme described in [1]. The transition from trapped mode to standing wave which occurs at one end of each of the branches is investigated in detail.
\end{abstract}

Keywords: embedded trapped modes; branch structure; cut-off; waveguide; standing wave

\section{Introduction}

In a previous paper McIver, Linton, McIver, Zhang \& Porter [1] proved that trapped modes exist for a countably infinite set of sufficiently long thin plates on the centreline of a twodimensional acoustic guide at wave numbers which are above the first cut-off for antisymmetric wave propagation down the guide. Trapped modes are localised oscillations which have finite energy and their existence in acoustic guides at wave numbers below the first antisymmetric cut-off has been well-documented (see for example [3], [4], [5], [6] and [7]). 
For wave numbers above the cut-off the eigenvalue associated with the trapped mode is said to be embedded in the continuous spectrum of the relevant operator. It is much harder to prove the existence of embedded trapped modes and they are known to be unstable to arbitrary perturbations in the body geometry. In general a perturbation in the obstacle or its position in the guide transforms the eigenvalue into a so-called 'complex resonance'. A description of such resonances for off-centre bodies in guides is given by Aslanyan, Parnovski \& Vassiliev [8]. However, in [1] it was shown numerically that for wave numbers between the first and second cut-off for antisymmetric wave propagation down the guide, an obstacle which supports an embedded trapped mode may be varied along a specific path within a two parameter family of geometric perturbations in such a way that the trapped mode persists. In particular we showed that if the flat plate aligned with the guide walls is deformed into an ellipse of a particular size and aspect ratio then the trapped mode remains and in fact a branch of trapped modes exists which starts with a plate on the centreline of the guide and terminates with a standing wave for a flat plate aligned perpendicular to the guide walls, and passes through a series of ellipses in-between. Other two parameter families of perturbations are possible and in [1] it was shown that a branch of modes exists for shapes of the form $x^{\nu}+y^{\nu}=a^{\nu}$, where $x$ is measured down the guide, $y$ is measured across the guide and $\nu$ and $a$ vary along the branch.

In the previous work we concentrated solely on branches of modes which emanate from the plate of smallest length on the centreline. However each of the plates is the starting point for a branch of modes and in this work we investigate the structure of the higher branches when the plates are deformed into either rectangular blocks or ellipses. Rectangular blocks are chosen because they have the interesting property that $\sin 3 \pi y / 2 d$ is a standing wave which can be supported by a block of half-depth $b=d / 3$, where $2 d$ is the width of the guide and $y$ is measured across the guide from the centreline, irrespective of the length of the block. It is found that one set of branches of trapped modes terminate in standing waves for blocks of this depth but that the others do not. Furthermore it is possible to obtain simple but accurate approximations to the trapped mode wave numbers from a two-term matched eigenfunction expansion and this procedure is used to obtain qualitative information about the structure of the branches.

The paper is organised as follows. In the next section the solution for the trapped mode potential for a rectangular block is written in terms of an eigenfunction expansion. The usual matching conditions are applied and the resulting series are truncated at two terms. 
This leads to a transcendental equation for the wave number nondimensionalised by the plate length. This approximation suggests that if $b / d<1 / 3$ then there is a countably infinite set of blocks which can support trapped modes. In $\S 3$ data is presented which shows good agreement between results obtained from this approximate solution and numerical results from the integral equation formulation described in [1]. The transcendental equation which arises from the approximate method is investigated in detail and information about the branch structure of the trapped modes is obtained. Results are also presented which illustrate the branch structure of trapped modes for ellipses and the differences between these and the branches for the rectangular blocks are analysed.

\section{Approximate trapped mode solution for a rectangular}

\section{block}

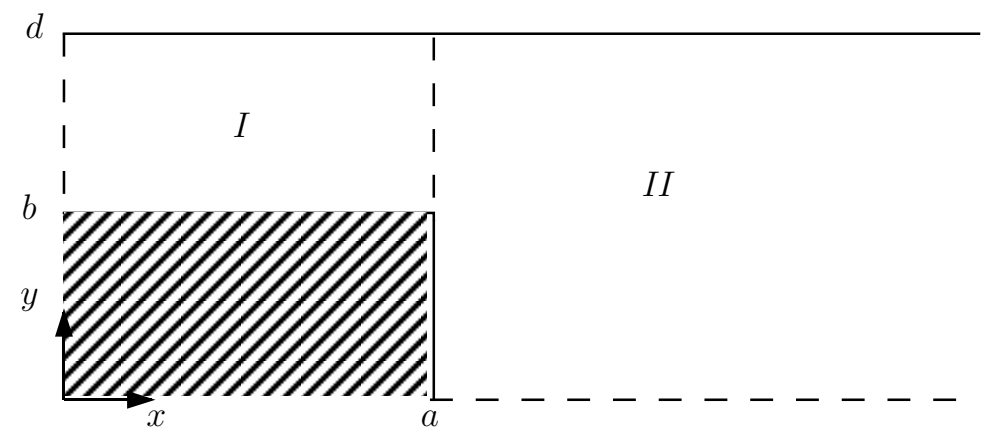

Figure 1: Definition sketch.

In this section an approximate solution for a trapped mode in the vicinity of a rectangular block is derived, at a frequency which is above the first cut-off for antisymmetric wave propagation down the guide but below the second such cut-off. A quarter section of the block and guide is illustrated in Figure 1 and a trapped mode potential is sought which is symmetric in $x$ and antisymmetric in $y$. A trapped mode which has this two-fold symmetry is sought as this means that there is only one type of wave which can propagate to either infinity. Moreover as the body is symmetric in $x$, there is only one condition which needs to be applied to ensure that the amplitudes of the waves propagating to both infinities are zero. It is expected that another set of modes exists that are antisymmetric in both $x$ and $y$, but it is not thought possible to relax the condition of antisymmetry in $y$. 
The trapped mode potential $\phi$ satisfies

$$
\left(\nabla^{2}+k^{2}\right) \phi=0
$$

in the guide exterior to the body, where $k^{2}=\omega^{2} / c^{2}$ and $\omega$ is the angular frequency of oscillation of the mode and $c$ is the speed of sound. The boundary conditions are given by

$$
\begin{gathered}
\phi=0 \quad \text { on } y=0, \quad x>a, \\
\frac{\partial \phi}{\partial y}=0 \quad \text { on } y=d, \quad x>0 \quad \text { and on } y=b, \quad 0<x<a, \\
\frac{\partial \phi}{\partial x}=0 \quad \text { on } \quad x=0, \quad b<y<d \quad \text { and on } x=a, \quad 0<y<b
\end{gathered}
$$

and

$$
\phi \rightarrow 0 \quad \text { as } \quad x \rightarrow \infty
$$

Evans, Levitin \& Vassiliev [4] proved that trapped modes exist for blocks of arbitrary length $a$ when $k d<\pi / 2$. The aim here is to seek blocks which support trapped modes for values of $k d$ in the range $\pi / 2<k d<3 \pi / 2$, the upper limit being the infimum of the set of values of $k d$ for which antisymmetric waves of the form $\mathrm{e}^{i \alpha x} \sin 3 \pi y / 2 d, 9 \pi^{2} / 4 d^{2}+\alpha^{2}=k^{2}$ can propagate down the guide. The formulation of the problem follows closely that described in Evans \& Linton [2]. The solution for $\phi$ is written as an eigenfunction expansion in each of regions $I$ and $I I$ and the coefficients in the expansion are determined by the requirement that the potential and velocity are continuous on the common boundary and that the horizontal velocity is zero on $x=a, 0<y<b$.

The potential in region $I$ is written as

$$
\phi(x, y)=\sum_{n=0}^{\infty} \frac{U_{n}^{(1)} \cosh k_{n} x}{k_{n} \sinh k_{n} a} \psi_{n}(y)
$$

where $\left\{U_{n}^{(1)}\right\}$ are the unknown coefficients and $\left\{\psi_{n}(y)\right\}$ is the complete orthonormal set of functions given by

$$
\psi_{n}(y)=\left(\frac{\epsilon_{n}}{d-b}\right)^{1 / 2} \cos p_{n}(d-y), \quad n=0,1, \ldots
$$

where

$$
\begin{gathered}
p_{n}=\frac{n \pi}{d-b}, \\
\epsilon_{n}= \begin{cases}1, & n=0 \\
2, & n \geq 1,\end{cases}
\end{gathered}
$$




$$
k_{0}=\mathrm{i} k, \quad k_{1}=\mathrm{i} q=\mathrm{i}\left(k^{2}-p_{1}^{2}\right)^{1 / 2}
$$

and

$$
k_{n}=\left(p_{n}^{2}-k^{2}\right)^{1 / 2}, n \geq 2 .
$$

As modes are sought in the range $\pi / 2<k d<3 \pi / 2$ and $p_{n} d \geq n \pi$ then $k_{n} d$ is real for $n \geq 2$. However if

$$
\frac{\pi}{(1-b / d)}<k d<\frac{3 \pi}{2}
$$

then $k_{1} d$ is purely imaginary and so from the series in (6) two wave-like terms may exist in the inner region. Such a pair of waves is only possible if (12) yields a non-zero range of values for $k d$, that is if $b / d<1 / 3$. From now on it will be assumed that $b / d$ lies below this critical value. It is interesting to note that at $b / d=1 / 3$ and $k d=3 \pi / 2$ the function

$$
\phi=\sin \frac{3 \pi y}{2 d}
$$

is a standing wave solution to (1) which satisfies the boundary conditions (2) - (4), irrespective of the length of the block.

The potential in region $I I$ is written as

$$
\phi(x, y)=-\sum_{n=2}^{\infty} \frac{U_{n}^{(2)}}{\kappa_{n}} \mathrm{e}^{-\kappa_{n}(x-a)} \Psi_{n}(y)
$$

where $\left\{U_{n}^{(2)}\right\}$ are the unknown coefficients and $\left\{\Psi_{n}(y)\right\}$ is the complete orthonormal set of functions given by

$$
\Psi_{n}(y)=\left(\frac{2}{d}\right)^{1 / 2} \sin l_{n}(d-y), \quad n=1,2, \ldots,
$$

where

$$
l_{n}=\frac{(2 n-1) \pi}{2 d}
$$

and

$$
\kappa_{n}=\left(l_{n}^{2}-k^{2}\right)^{1 / 2}, n \geq 2 .
$$

It should be noted that, as $k d>\pi / 2$, a possible term which multiplies $\Psi_{1}(y)$ contains progressive waves and so its coefficient is forced to be zero by the requirement that $\phi \rightarrow 0$ as $x \rightarrow \infty$.

Continuity of the horizontal component of the velocity on $x=a$ gives

$$
\sum_{n=2}^{\infty} U_{n}^{(2)} \Psi_{n}(y)= \begin{cases}\sum_{n=0}^{\infty} U_{n}^{(1)} \psi_{n}(y), & b<y<d \\ 0, & 0<y<b\end{cases}
$$


Multiplication of both sides of (18) by $\Psi_{m}(y), m=1,2, \ldots$ and integration over $[0, d]$ gives

$$
\sum_{n=0}^{\infty} U_{n}^{(1)} c_{n m}= \begin{cases}U_{m}^{(2)}, & m=2,3, \ldots \\ 0, & m=1\end{cases}
$$

where

$$
c_{n m}=\int_{b}^{d} \psi_{n}(y) \Psi_{m}(y) \mathrm{d} y .
$$

Continuity of potential on $x=a, b<y<d$ gives

$$
\sum_{n=0}^{\infty} \frac{U_{n}^{(1)}}{k_{n}} \operatorname{coth} k_{n} a \psi_{n}(y)=-\sum_{n=2}^{\infty} \frac{U_{n}^{(2)}}{\kappa_{n}} \Psi_{n}(y), \quad b<y<d
$$

Multiplication of both sides of $(21)$ by $\psi_{m}(y), m=0,1, \ldots$ and integration over $[b, d]$ gives

$$
\frac{U_{m}^{(1)}}{k_{m}} \operatorname{coth} k_{m} a=-\sum_{n=2}^{\infty} \frac{U_{n}^{(2)}}{\kappa_{n}} c_{m n}, \quad m=0,1, \ldots
$$

A trapped mode solution for which $\pi / 2<k d<3 \pi / 2$ corresponds to a nontrivial solution of the systems of equations in (19) and (22). A crude approximation to these equations is obtained by truncating the expansion in region $I$ at $n=1$ and the expansion in region $I I$ at $n=2$. Physically this corresponds to allowing for the effects of the two possible wave-like terms in the inner region and the leading order non-zero term in the outer region. This procedure yields the matrix system of equations

$$
A \mathbf{u}=\left(\begin{array}{ccc}
c_{01} & c_{11} & 0 \\
c_{02} & c_{12} & -1 \\
-k^{-1} \cot k a & 0 & \kappa_{2}^{-1} c_{02} \\
0 & -q^{-1} \cot q a & \kappa_{2}^{-1} c_{12}
\end{array}\right)\left(\begin{array}{c}
U_{0}^{(1)} \\
U_{1}^{(1)} \\
U_{2}^{(2)}
\end{array}\right)=\left(\begin{array}{l}
0 \\
0 \\
0 \\
0
\end{array}\right) .
$$

This system has a non-trivial solution if and only if $\operatorname{rank}(A) \leq 2$. The matrix $A$ depends on the three non-dimensional parameters $b / d, a / d$ and $k d$ but the coefficients $c_{n m}$ only depend on $b / d$. As the first two rows of the matrix are independent of $a / d$ and $k d$, it is convenient to express the condition that $\operatorname{rank}(A) \leq 2$ as the two equations

$$
\left|\begin{array}{ccc}
c_{01} & c_{11} & 0 \\
c_{02} & c_{12} & -1 \\
-k^{-1} \cot k a & 0 & \kappa_{2}^{-1} c_{02}
\end{array}\right|=0
$$

and

$$
\left|\begin{array}{ccc}
c_{01} & c_{11} & 0 \\
c_{02} & c_{12} & -1 \\
0 & -q^{-1} \cot q a & \kappa_{2}^{-1} c_{12}
\end{array}\right|=0 .
$$


These equations are then expanded and after manipulation, combination with (10) and (17) and evaluation of the integrals $c_{n m}$ they reduce to a transcendental equation for $k a$ for a given value of $b / d$, namely

$$
f(k a, b / d)=k a \tan k a-c q a \tan q a=0,
$$

where

$$
q a=k a\left[1-\frac{4}{9(1-b / d)^{2}}-\frac{262144 \tan ^{2} k a \cos ^{4}(3 \pi b / 2 d)}{729 \pi^{4}(1-b / d)^{4}(5-3 b / d)^{2}(1-3 b / d)^{2}}\right]^{1 / 2}
$$

and

$$
c=\frac{18(1-b / d)^{4}}{(3-b / d)(1+b / d)(5-3 b / d)(1-3 b / d)} .
$$

Thus $c$ just depends on $b / d$ and as $b / d<1 / 3, c$ is positive. Once $k a$ has been determined from (26) the remaining non-dimensional parameters may be written in terms of it. In particular it may be shown that

$$
\begin{gathered}
\frac{a}{d}=\frac{2 k a}{3 \pi}\left[1+\frac{65536 \tan ^{2} k a \cos ^{4}(3 \pi b / 2 d)}{81 \pi^{4}(1-b / d)^{2}(5-3 b / d)^{2}(1-3 b / d)^{2}}\right]^{1 / 2}, \\
k d=\frac{k a}{a / d}=\frac{3 \pi}{2}\left[1+\frac{65536 \tan ^{2} k a \cos ^{4}(3 \pi b / 2 d)}{81 \pi^{4}(1-b / d)^{2}(5-3 b / d)^{2}(1-3 b / d)^{2}}\right]^{-1 / 2},
\end{gathered}
$$

and

$$
\kappa_{2} a=\frac{256 \cos ^{2}(3 \pi b / 2 d)}{9 \pi^{2}(1-b / d)(5-3 b / d)(1-3 b / d)} k a \tan k a .
$$

The potential in region $I I$ is formed from a sum of decaying exponentials, and so from (14) $\kappa_{2} a$ must be positive. Thus from (31) as $b / d<1 / 3$, tan $k a$ must be positive, that is

$$
n \pi<k a<(n+1 / 2) \pi, \quad n=0,1, \ldots
$$

From (26) and (32) qa tan qa must be positive, which means that qa must be real and so from (6) and (10) there are two waves in the inner region. Furthermore as qa is chosen to be the positive square root in (10) and $q a \tan q a>0$ then $\tan q a>0$, that is

$$
m \pi<q a<(m+1 / 2) \pi, \quad m=0,1, \ldots .
$$

The pair of integers $(n, m)$ measure the number of wavelengths of the modes $\cos k x$ and $\cos q x \cos [\pi(d-y) /(d-b)]$ respectively, which fit in the inner region and it is convenient to identify the roots of (26) by these numbers. From (27), (32) and (33)

$$
m \pi<q a<\frac{\sqrt{5}}{3} k a<\frac{\sqrt{5}}{3}(n+1 / 2) \pi
$$

and this restricts the possible values of $m$ to integers such that

$$
0 \leq m<\frac{\sqrt{5}}{3}(n+1 / 2)
$$




\section{Results and discussion}

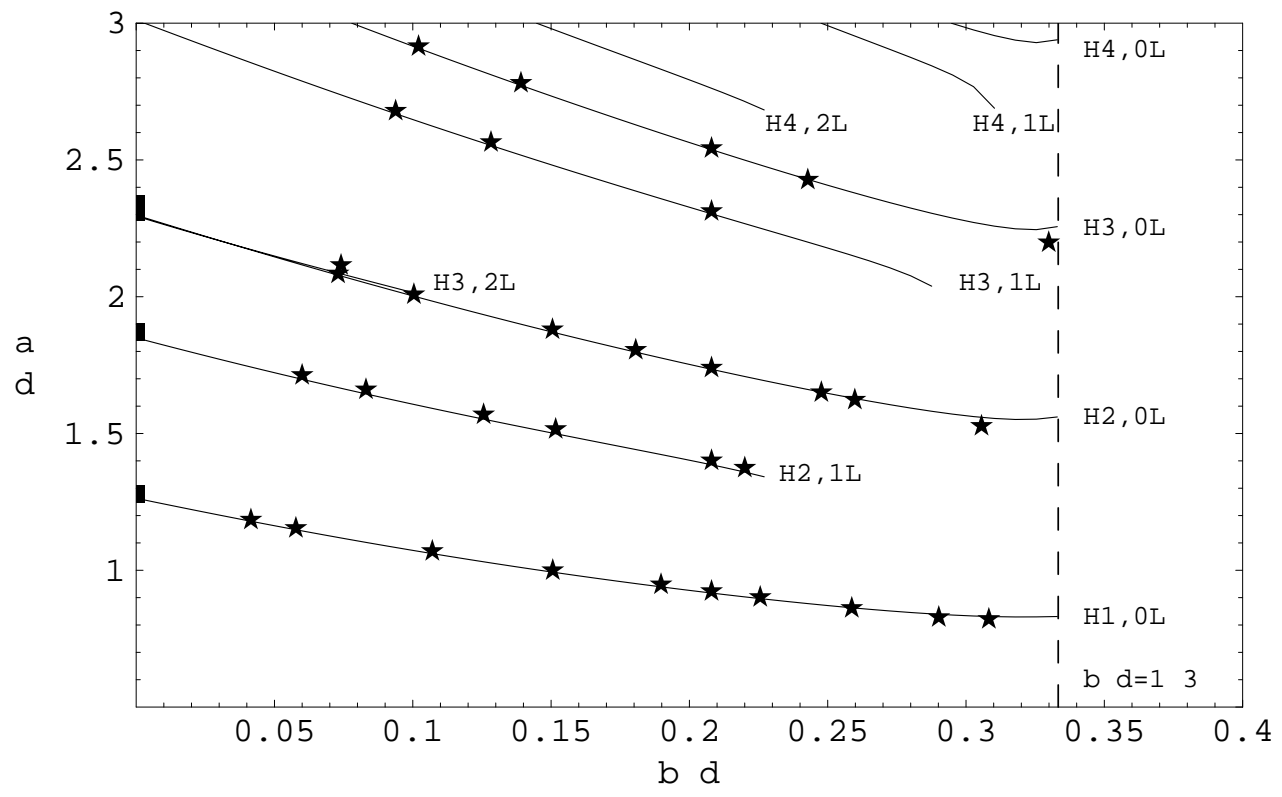

Figure 2: Lengths of blocks that are able to support trapped modes; —values from transcendental equation; $\mathbf{m}$ : long plate approximation; $\star$ : numerical values from integral equation.

Suppose that we want to determine all blocks that support trapped modes which have a half-length $a<a^{*}$ where $a^{*}$ is some given value. As $k d$ lies in the range $\pi / 2<k d<3 \pi / 2$, $k a<k a^{*}=(k d)\left(a^{*} / d\right)<3 \pi a^{*} / 2 d$. From (32) as $k a<(n+1 / 2) \pi$ for some integer $n$, if all the roots of the transcendental equation (26) are found in the range $0<k a<\pi\left(\left[3 a^{*} / 2 d\right]+1 / 2\right)$, where [.] denotes integer part, then all blocks with $a<a^{*}$ that support trapped modes will have been found. Figure 2 illustrates all such blocks with $a / d<3$ as a function of $b / d$ and figures 3 and 4 give the corresponding wave numbers $k d$ as functions of $b / d$ and $a / d$. The lines indicate values calculated from the transcendental equation (26) and the stars are the numerical results from the integral equation method described in [1]. This latter method becomes time-consuming to run numerically as $n$ increases and so it was only used to calculate trapped modes on branches for which $n \leq 3$.

There is close agreement between the approximate and full numerical results and so by analysing the transcendental equation in (26) some important information about the behaviour of the trapped modes may be determined. First it should be noted that the approximate method yields the same number of branches of trapped modes as the exact method and indeed was instrumental in determining that in the $a / d-b / d$ plane there are 


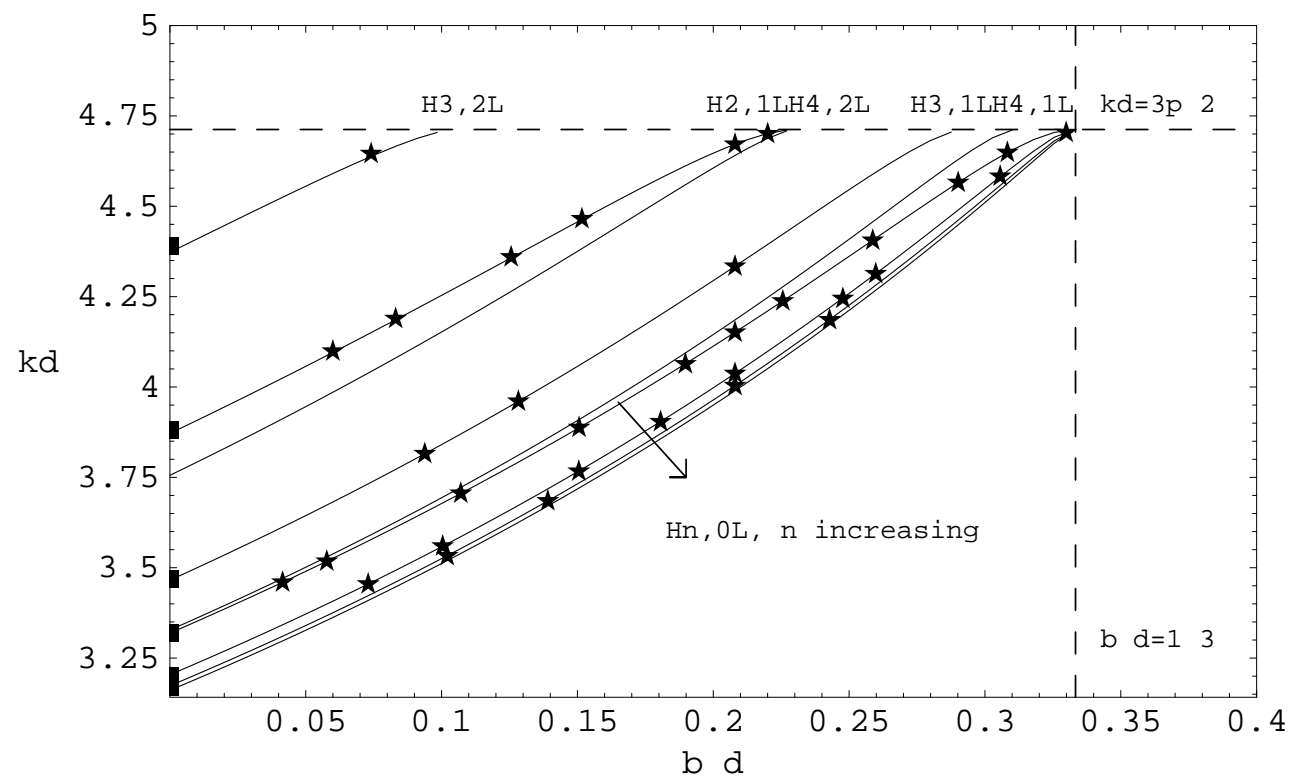

Figure 3: Trapped mode wave numbers for blocks as a function of $b / d$; - : approximate values from transcendental equation; $\mathbf{m}$ : long plate approximation; $\star$ : numerical values from integral equation.

two branches of modes very close together, namely the branches $(3,2)$ and $(2,0)$. This means that there are two sets of blocks with very similar sizes that support trapped modes, but as may be seen in figure 3 the wave numbers of these modes are very different. Although the bound on the number of branches given in (35) is correct, it is not tight as it permits the branches $(1,1)$ and $(4,3)$, neither of which are found numerically. When $b / d=0$ the rectangular block reduces to a plate on the centreline of the guide. Approximations to the values of $a / d$ and $k d$ for these plates were derived in [1] from a long plate analysis. The long plate results are denoted by $\mathbf{m}$ on each of the figures and are seen to agree well with the other numerical results.

From figure 3 it is clear that the other ends of all the branches occur when $k d$ reaches the second cut-off, $3 \pi / 2$. However, from figure 2 the size of the block at which this happens depends on the type of branch of modes. For all the branches of modes designated by $(n, 0), b / d=1 / 3$ at the terminating point and, as has already been observed, the potential $\phi=\sin 3 \pi y / 2 d$ is a standing wave with $k d=3 \pi / 2$ for any block of this depth irrespective of its length. Thus the trapped modes on these branches tend to this standing wave, and in fact the whole of the line $b / d=1 / 3$ in figure 2 corresponds to standing wave solutions. In 


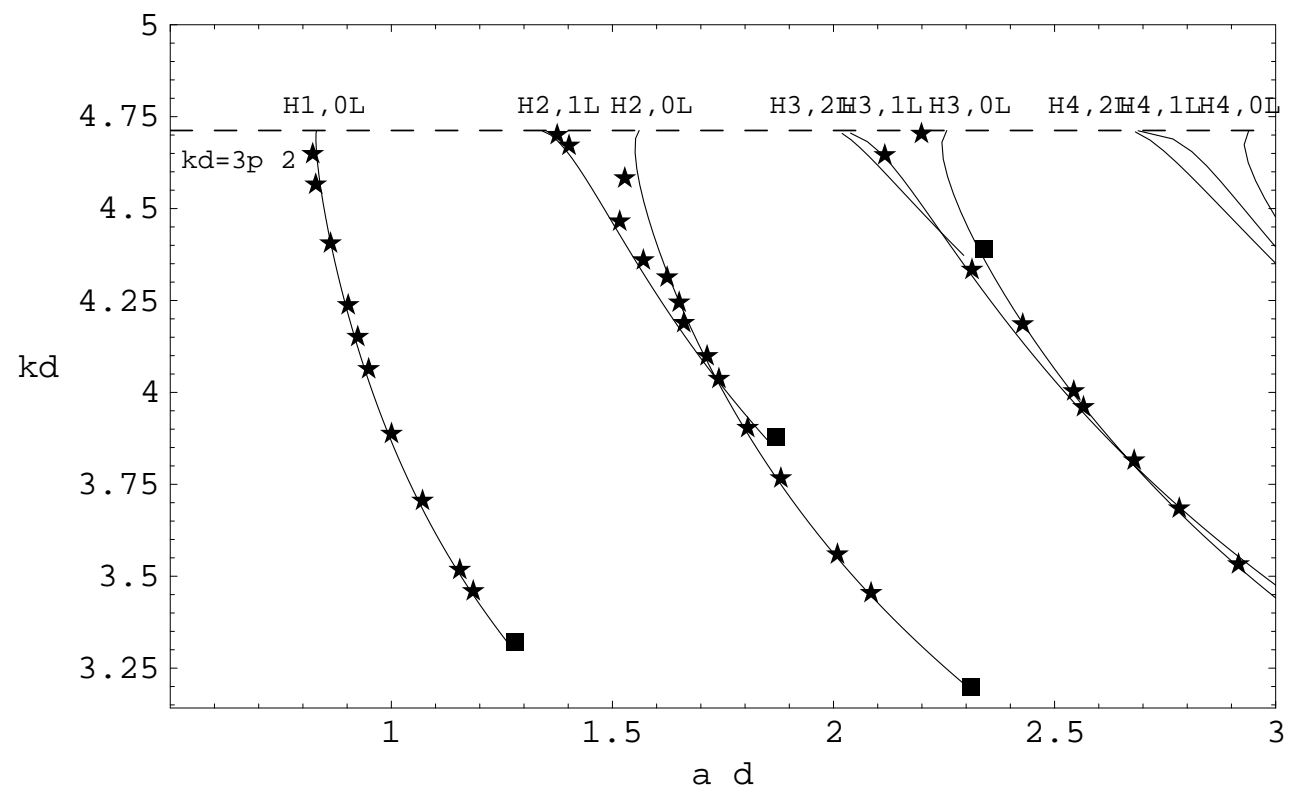

Figure 4: Trapped mode wave numbers for blocks as a function of $a / d$; - : approximate values from transcendental equation; $\mathbf{m}$ : long plate approximation; $\star$ : numerical values from integral equation.

the limit as $b / d \rightarrow 1 / 3$ it may be shown that the transcendental equation (26) reduces to

$$
\tan k a-\frac{k a}{4}=0
$$

and from (29) the limiting values of $a / d$ are then given by $a / d=2 k a / 3 \pi$.

The branches designated by $(n, m), m \neq 0$, do not terminate at $b / d=1 / 3$ and so from (30) $\tan k a \rightarrow 0$ as $k d \rightarrow 3 \pi / 2$. Thus from (32) $k a \rightarrow n \pi$ and from (29) $a / d \rightarrow 2 n / 3$. Moreover from (26) and (27)

$$
\tan q a \rightarrow \tan \left\{k a\left[1-\frac{4}{9(1-b / d)^{2}}\right]^{1 / 2}\right\} \rightarrow 0
$$

which from (33) gives $q a \rightarrow m \pi$ and from (37) the limiting values of $b / d$ as

$$
\frac{b}{d}=1-\frac{2}{3\left(1-m^{2} / n^{2}\right)^{1 / 2}}
$$

Thus for a fixed value of $n$ and $m \neq 0$, these branches all terminate at the same value of $a / d$ when $k d=3 \pi / 2$. This is most easily seen from the results in figure 4 . In these cases the limiting standing wave is no longer the simple function $\phi=\sin 3 \pi y / 2 d$, as this does not satisfy the boundary conditions on the block. The standing wave asymptotes to this function as $x \rightarrow \pm \infty$ but is modified in the neighbourhood of the block. The limiting value of $b / d$ given by (38) also demonstrates why neither of the branches $(1,1)$ nor $(4,3)$ were observed 
numerically, as the values of $b / d$ at the ends of their branches would have to be given by $-\infty$ and -0.0079 respectively, both of which are clearly unphysical.

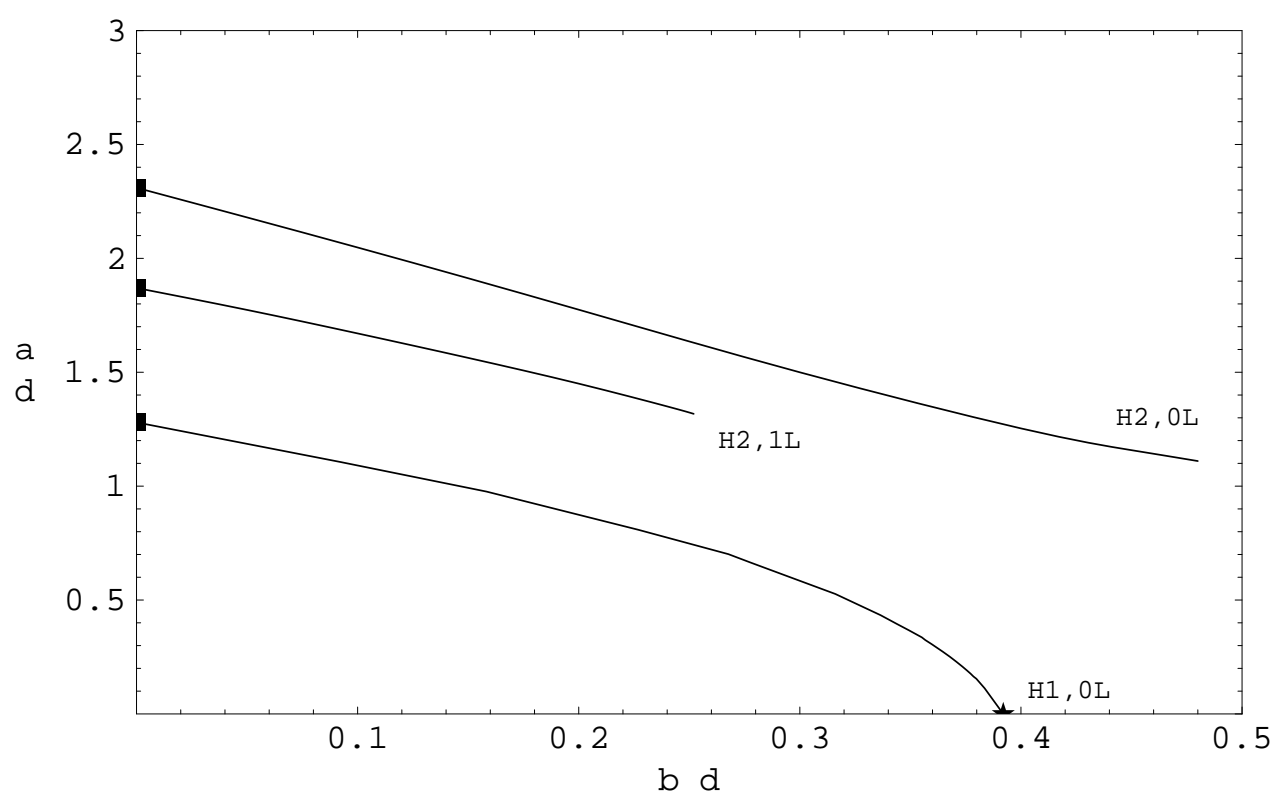

Figure 5: Lengths of ellipses that are able to support trapped modes; $\mathbf{m}$ : long plate approximation; $\star$ : perpendicular plate; ——: numerical values from integral equation.

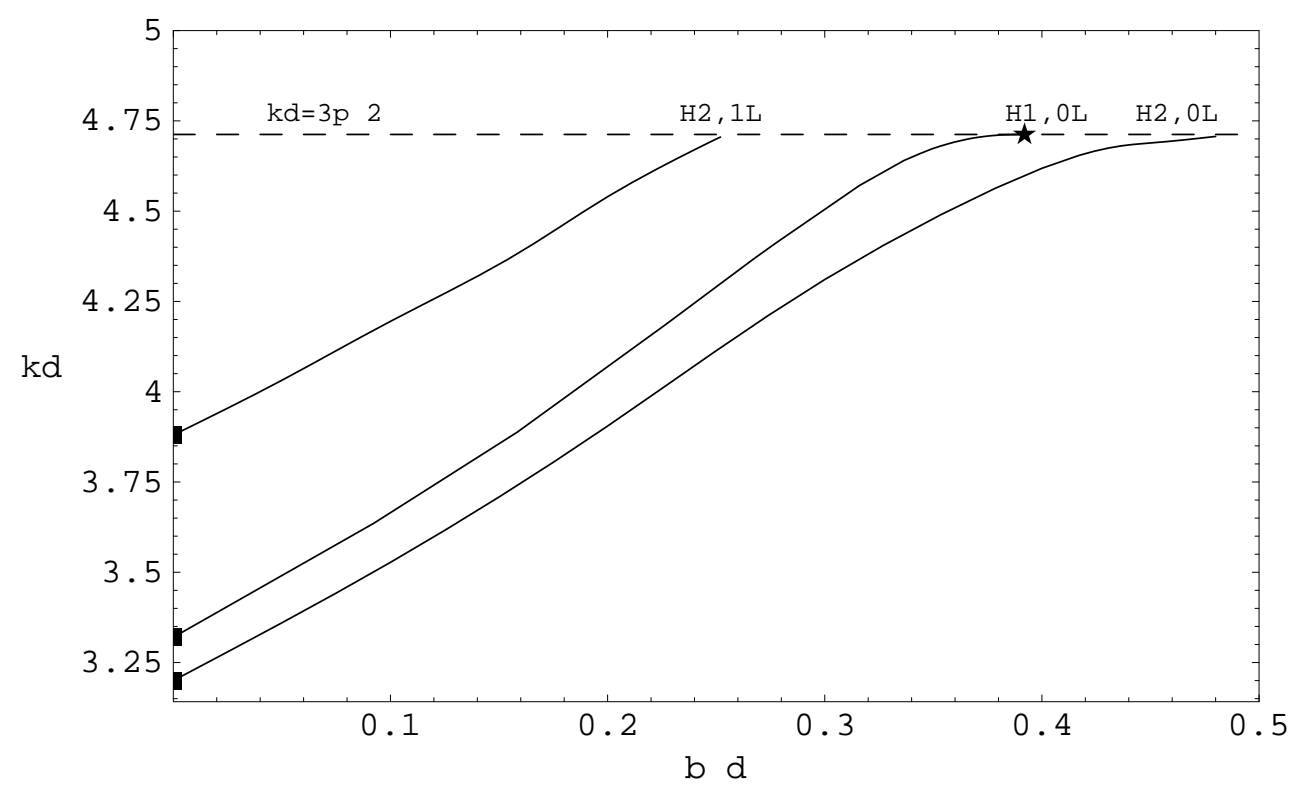

Figure 6: Trapped mode wave numbers for ellipses; $\mathbf{-}$ : long plate approximation; : perpendicular plate; ——: numerical values from integral equation.

Figures 5, 6 and 7 show the structure of the first three branches of trapped modes for ellipses of aspect ratio $b / a$ situated on the centreline of the guide. The results are all obtained 


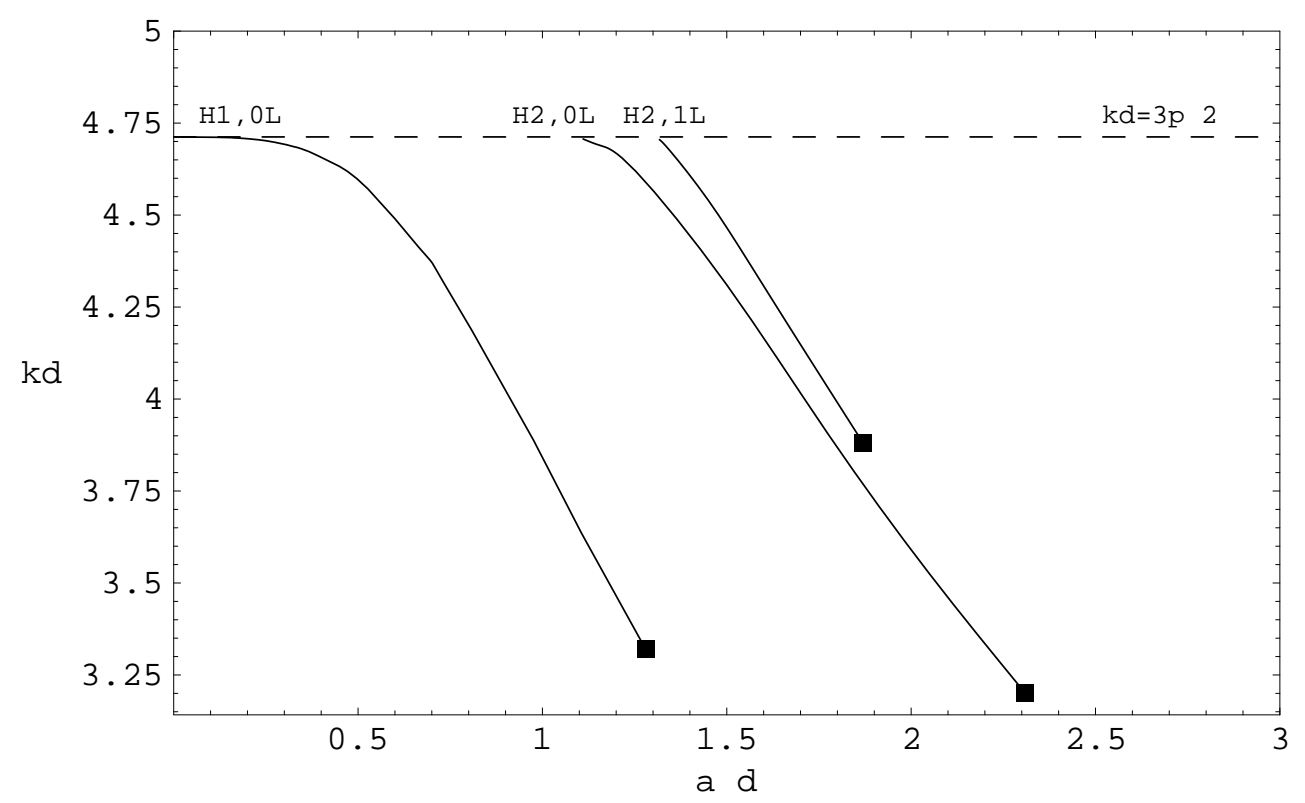

Figure 7: Trapped mode wave numbers for ellipses; a: long plate approximation; ——: numerical values from integral equation.

from the integral equation method described in [1] apart from the values for $b / d=0$ which come from the long plate approximation and the other end of the lowest branch which is a limiting value for a plate perpendicular to the guide walls. A comparison of these results and those for the blocks in figures 2, 3 and 4 shows that instead of terminating at a non-zero value of $a / d$, the lowest branch for the ellipse terminates at $a / d=0$. The value of $b / d$ at this point was derived in [1] from a slender body theory and is given by $b / d=B$, where $B$ is the root of the transcendental equation $J_{1}(2 \pi B)-J_{1}(\pi B)=0(B \approx 0.392)$ and $J_{1}$ denotes the first-kind Bessel function of order one. It was shown in [1] that the lowest branch for the vast majority of obstacles terminates at $a / d=0$ and the limiting value of $b / d$ depends on the precise shape of the body as it approaches this limit. This is because an obstacle for which $a / d=0$ corresponds to a plate which is perpendicular to the guide walls and such a plate supports the standing wave $\phi=3 \pi y / 2 d$ irrespective of the value of $b / d$. It is the rectangular block which is the special geometry that can support this standing wave for an arbitrary value of $a / d$ when $b / d=1 / 3$.

From figure 6 the second and third branches for the ellipse terminate at $k d=3 \pi / 2$ in a similar fashion to those for the block. For convenience all the branches for the ellipse are labelled in the same way as those for the block even though there are some differences in behaviour. For example they all terminate at different values of $b / d$ from each other, and in particular the branch labelled $(2,0)$ attains a higher value of $b / d$ than does the branch 
$(1,0)$. The limiting values of $b / d$ on this and higher branches have to be found numerically as it is not possible to determine analytically which ellipses with finite, non-zero area can support standing waves. Furthermore the branch structure for the trapped modes for the ellipse is expected to be typical of that for the vast majority of symmetric obstacles which may be deformed from flat plates, whilst the rectangular block is a special body which has certain distinctive properties.

\section{Conclusion}

In this work we have investigated the structure of branches of embedded trapped modes which exist when obstacles with a two-fold symmetry are placed on the centreline of a two-dimensional acoustic waveguide. The modes are antisymmetric about the centreline of the channel and symmetric about the other plane of symmetry and have frequencies that are between the first and second cut-off for antisymmetric wave propagation down the guide. The particular geometries considered here were rectangular blocks and ellipses, although the numerical scheme developed in [1] is applicable to many other geometrical shapes. A two-term matched eigenfunction expansion was used to show that a countably infinite set of branches of trapped modes exist for a rectangular block and that each branch may be labelled by the pair of integers $(n, m)$, where the possible values of $m$ and $n$ satisfy $0 \leq m<\sqrt{5}(n+1 / 2) \pi / 3$. A similar branch labelling was used for the ellipse and for both types of bodies each branch of trapped modes starts with a trapped mode for a thin plate on the centreline of the guide and terminates with a standing wave. The standing wave asymptotes to the function $\phi=\sin 3 \pi y / 2 d$ as $x \rightarrow \pm \infty$, but may be modified in the neighbourhood of the obstacle to take account of the body boundary conditions.

\section{Acknowledgements}

The work of J. Zhang was supported by EPSRC grant GR/M30937

\section{References}

[1] McIver, M., Linton, C. M., McIver, P., Zhang, J. \& Porter, R. 2000. Embedded trapped modes for obstacles in two-dimensional waveguides. To appear in Quarterly J. Mechanics Applied Mathematics. 
[2] Evans, D. V. \& Linton, C. M. 1991. Trapped modes in open channels J. Fluid Mechanics 225, 153-175.

[3] Evans, D. V., Linton, C. M. \& Ursell, F. 1993. Trapped mode frequencies embedded in the continuous spectrum Quarterly J. Mechanics Applied Mathematics 46, 253-274.

[4] Evans, D. V., Levitin, M. \& Vassiliev, D. 1994. Existence theorems for trapped modes. J. Fluid Mechanics 261, 21-31.

[5] Davies, E.B. \& Parnovski, L. 1998. Trapped modes in acoustic waveguides. Quarterly J. Mechanics Applied Mathematics 51,477-492.

[6] Groves, M.D. 1998. Examples of embedded eigenvalues for problems in acoustic waveguides Mathematical Methods in the Applied Sciences 21(6), 479-488.

[7] Khallaf, N. S. A., Parnovski, L. \& Vassiliev, D. 2000. Trapped modes in a waveguide with a long obstacle. J. Fluid Mechanics 403, 251-261.

[8] Aslanyan,A., Parnovski, L. \& Vassiliev, D. 2000. Complex resonances in acoustic waveguides. Quarterly J. Mechanics Applied Mathematics 53, 429-447. 